\title{
La culture constitutionnelle du peuple. Une synthèse.
}

\author{
Xavier Magnon \\ Professeur de droit public \\ Aix Marseille Univ, Université de Toulon, Univ Pau \& Pays Adour, \\ CNRS, DICE, ILF, Aix-en-Provence, France
}

Le sujet est original, et même, à la mode, comme certains ont pu l'affirmer ${ }^{1}$; il est incontestablement un sujet d'étude qui n'est pas familier aux juristes. «La culture constitutionnelle du peuple » n'est pas un objet d'analyse saisi par ces derniers. Aussi ce sujet fournit-il aux juristes l'occasion de s'interroger sur la connaissance qu'ils sont susceptibles de produire sur un objet factuel qui constitue, par ailleurs, un sujet sociétal contemporain.

Si l'on tente, dans un premier temps, de retenir quelques sens minimums pertinents du terme de culture, l'on peut s'interroger avec A.-M. Le Pourhiet pour savoir si le terme désigne un savoir, un babitus ou un comportement. Des sens communs mobilisables, il faut sans doute retenir la culture comme désignant des "connaissances dans un domaine particulier» ou comme un «ensemble des phénomènes matériels et idéologiques qui caractérisent un groupe ethnique ou une nation, une civilisation, par opposition à un autre groupe ou à une autre nation $»^{2}$. De manière plus précise, L. M. Friedman identifie la «culture juridique » comme englobant «la connaissance, les attitudes et les modèles de comportement du public à l'égard du système juridique $»^{3}$.

Lorsqu'elle porte sur la Constitution, l'étude de la culture présente un intérêt tout à fait déterminant dans la discipline du droit constitutionnel. Alors que la Constitution est une norme d'encadrement de l'exercice du pouvoir et donc un instrument juridique de limitation de celui-ci, la culture constitutionnelle participe de cette même lutte d'encadrement et de résistance face au pouvoir, comme cela a pu être souligné par M. Stéfanini. Plus la culture constitutionnelle est importante au sein du peuple et plus celui-ci est en mesure d'apprécier l'exercice du pouvoir et d'en identifier les abus ou les dérives éventuelles. Selon une lecture politico-sceptique, il peut être utile aux dominants que ne se développe pas une culture constitutionnelle ou que celle-ci demeure réservée à une élite. Dans les deux cas, l'absence de culture constitutionnelle profite au pouvoir. A cet égard, R. H. Mohamed a rappelé que la limitation de la culture constitutionnelle pouvait servir le pouvoir et, en particulier, une culture constitutionnelle ciblée. En tout état de cause, J. Vachey a pu constater que le peuple, en général, ne connait pas la Constitution ce qui constitue un obstacle au développement d'une culture constitutionnelle.

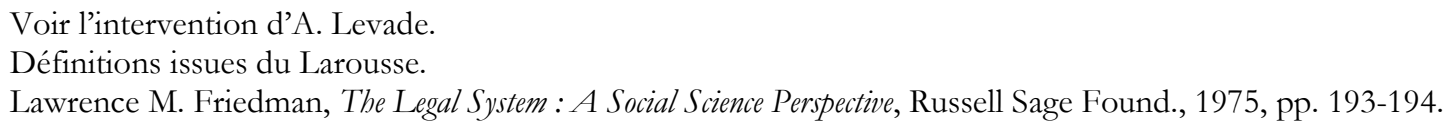


La question de la culture constitutionnelle est encore importante dans la mesure où la place du peuple est décisive dans les processus d'adoption et de modification des Constitutions, tout comme d'ailleurs dans les révolutions politiques visant à renverser l'ordre constitutionnel établi. P. Alvazzi Del Frate a pu souligner, en particulier, que, parfois, face à des difficultés de modification de la Constitution, seule une révolution et donc une rupture avec l'ordre constitutionnel peut être entreprise. A l'occasion de telles ruptures, la place du peuple est déterminante. Le peuple occupe ainsi une place décisive dans les processus constituants, de révision constitutionnelle et de révolution constitutionnelle. Dans le prolongement, A. Levade a souligné combien la Constitution trouvait sa légitimité dans le peuple, ce qui témoigne de toute la pertinence pour celui-ci de pouvoir disposer d'une culture constitutionnelle.

Malgré l'importance du sujet pour les constitutionnalistes, il n'en demeure pas moins qu'il leur est difficilement saisissable, dans la mesure où le sujet mobilise des données d'ordre factuelles. Saisir les comportements des acteurs du droit constitutionnel, et, surtout du juge ${ }^{4}$, de manière systématique ne pose pas de difficulté particulière. Le comportement du juge est saisissable, en partie, en tant que norme et en tant que comportement objectif, par le texte du jugement ou de l'arrêt. En revanche, face à des données purement factuelles, le juriste ne dispose pas de méthode d'analyse pertinente, de recueil comme de traitement de ces données. Il n'est d'ailleurs pas certain non plus que les juristes mobilisent des données ou des études produites par d'autres sciences sociales, ce qui serait, en l'occurrence, un moyen de contourner l'obstacle méthodologique.

Quoi qu'il en soit, l'étude de données factuelles par les juristes soulève une question d'ordre méthodologique. Sous l'angle juridique, J. Vachey a pu souligner le manque de données empiriquement observables permettant de saisir la culture constitutionnelle, du moins l'absence de données récentes. P. Alvazzi Del Frate a précisé que les cahiers de doléance(s) de 1789 constituent des éléments importants pour apprécier la culture constitutionnelle du peuple. Le «Grand débat national », en tant que réaction au mouvement des gilets jaunes, s'est inscrit, d'une certaine manière, dans cette logique de doléances. Il est ainsi possible de rechercher, en particulier, l'usage du terme «Constitution», même s'il est difficile d'en avoir une approche empirique systématique. En science politique, le même constat a été dressé par B. François, les données recueillies et observées datent des années 40 et 70, l'étude la plus récente a été entreprise en 2002.

\footnotetext{
$4 \quad$ Pour être plus précis, une approche systématique de la jurisprudence ne saurait être possible qu'à partir d'une recherche exhaustive de tous les jugements ou arrêts rendus par une juridiction, ce qui peut, parfois, limiter une étude systématique de la jurisprudence à celles de cours suprêmes ou de juges constitutionnels. Le comportement d'autres acteurs, tels par exemple, les parlementaires, s'avère déjà plus délicat à saisir de manière systématique, autant d'un point de vue quantitatif que qualitatif. Rien n'empêche, évidement, de procéder à des études à partir d'échantillons, à conditions de maitriser les méthodes et les techniques qui doivent être mobilisées en ce sens.
} 
Ce qui peut apparaitre, pour le moins, inquiétant est que, ces études, toutes anciennes qu'elles soient, montrent que le sujet est, pour le dire de manière quelque peu radicale, sans objet. B. François l'a rappelé. Selon les études empiriques sur le sujet, il n'existe pas de connaissance satisfaisante de la Constitution par le peuple. De plus, B. François a explicité le véritable objet "culture constitutionnelle». Il ne faut en effet pas entendre la "culture constitutionnelle» comme se rapportant à une question de connaissances, savantes, mais plutôt à ce qu'il convient de qualifier de compétences constitutionnelles. En effet, s'il n'existe pas de connaissance constitutionnelle du peuple, il existe, malgré tout, des compétences constitutionnelles, c'est-à-dire une conscience politique en dehors des processus de connaissances savantes, des «compétences pratiques » selon l'expression de P. Bourdieu.

L'on pourrait ajouter, également, mais nous y reviendrons, qu'il existe une représentation de la Constitution, et donc du droit constitutionnel par le peuple. Plus précisément, sans doute, dans l'appréhension de l'objet de ce colloque par les juristes, il est certes question de représentation, mais de la représentation par les juristes de ce qu'est la culture constitutionnelle du peuple, non pas de la représentation par le peuple de la Constitution. Sans doute avons-nous là l'objet tel qu'il peut être saisi par les juristes : une représentation savante, élaborée sans données empiriques observées, de la culture constitutionnelle du peuple. L'objet factuel est ainsi abordé, non pas, en lui-même, à partir de ce qu'il est grâce à des données empiriques observables, mais de la représentation qu'il est possible d'en avoir, à partir de l'ensemble des connaissances savantes du juriste, en l'occurrence, pour le sujet abordé aujourd'hui, celles des juristes constitutionnalistes. Les connaissances savantes d'une discipline façonnent l'objet, tout en se tenant éloignées de ce qu'il est, effectivement, dans la pratique.

Dans cette perspective, il est possible de développer un discours constructif, identifiant ce que devrait être le peuple dans une démocratie et défendant, en conséquence, ce que devrait alors être, en fonction de cet idéaltype de peuple, la culture constitutionnelle de celui-ci. La conception de la culture constitutionnelle ainsi adoptée serait en conséquence le reflet de ce que l'on considère comme nécessaire pour un bon fonctionnement de la démocratie. Il s'agirait de construire un modèle de culture constitutionnelle, en dehors de toute donnée empirique, susceptible de servir de référent à partir duquel l'on pourra identifier, imaginer et proposer les moyens matériels propres, en particulier, à développer la culture constitutionnelle.

Le peuple peut être ainsi «construit», en tant que personnage conceptuel, selon l'expression de G. Deleuze, à partir de la culture constitutionnelle qu'il devrait avoir parce qu'il est celui à qui s'adresse la Constitution. Les interrogations de J.-P. Dérosier sur ce qu'est «le peuple» font encore écho à cette réflexion.

Dans un tel contexte, deux questions semblent devoir se poser. Il faut, tout d'abord, s'attarder sur la question du sens et préciser et expliciter ce que l'on entend par «culture constitutionnelle» 
( $(\mathbb{I})$; avant, ensuite, de synthétiser ce que l'on peut dire de cette culture pour tenter de développer une culture constitutionnelle pour le peuple (\$ II).

\section{\ I - De quoi parle-t-on ? Qu'est-ce que la « culture constitutionnelle »?}

L'indétermination relative autour de l'objet d'étude «culture constitutionnelle » justifie que l'on revienne sur l'identification de cet objet. Deux perspectives peuvent être retenues pour l'éclairer. Selon une logique interne, la culture constitutionnelle peut se faire par la Constitution (A). Il ne s'agit pas d'apprécier ce qu'est la culture constitutionnelle mais, plutôt, de mettre en évidence comment elle se nourrit. Selon une logique externe, la culture constitutionnelle peut porter sur la Constitution (B) et il sera plutôt question ici d'apprécier ce qu'est la culture constitutionnelle.

$A$ - Une logique interne : la culture constitutionnelle par le droit constitutionnel

Le droit constitutionnel, le texte constitutionnel, peut, lui-même, être à l'origine du développement d'une culture constitutionnelle. La participation du peuple au processus constituant comme au processus de révision constitutionnelle favorisent une connaissance par celui-ci du texte sur lequel il est amené à se prononcer, qu'il s'agisse du texte constitutionnel dans son ensemble ou seulement d'une partie de celle-ci. Au regard des analyses de sciences politiques déjà évoquées, l'on ne saurait se faire trop d'illusions de ce que, au cours de tels processus, chaque citoyen prenne connaissance effectivement des textes sur lesquels il se prononce et s'informe sur le sens de ceux-ci, mais, au moins, peut-on supposer qu'un minimum de débat public conduise à informer les citoyens sur la Constitution. Autrement dit, faire participer le peuple à l'adoption ou à la modification de la Constitution constitue une occasion de générer ou de développer la culture constitutionnelle du peuple. Comme l'a évoqué P. Alvazzi Del Frate, lorsque que le peuple est à l'origine de la Constitution, lorsqu'il participe à la rédaction même du texte constitutionnel, quelles qu'en soient les modalités, il mobilise une culture constitutionnelle, quelle qu'en soit la qualité. Rafsandjani Hassani Mohamed a rappelé, qu'en Afrique, le peuple est fréquemment constituant. En effet, les processus d'adoption de la Constitution font souvent intervenir le peuple, en tant qu'instrument de légitimation. Dans le même sens, M. Baudrez a ajouté que la votation de la Constitution, en elle-même, participe de la culture constitutionnelle. J.-P. Dérosier a enfin rappelé que le texte constitutionnel renvoyait lui-même au peuple dans ses énoncés dispositionnels : les peuples sont visés par les Constitutions, le peuple devenant alors un objet du droit constitutionnel.

\section{B - Une logique externe : la culture constitutionnelle sur le droit constitutionnel}

La culture constitutionnelle peut prendre plusieurs formes.

Il a ainsi été question d'un "catéchisme constitutionnel», P. Alvazzi Del Frate a rappelé celui proposé en 1793 par Adrien Richer, sans qu'aucun ne défende la nécessité de prêcher un catéchisme à 
destination du peuple. A.M. Le Pourhiet, J.-P. Dérosier ont explicitement rejeté toute défense d'une culture constitutionnelle officielle. Comme l'a fait remarquer X. Philippe pour l'Afrique du Sud, il existe des organismes privés qui développent une culture constitutionnelle militante. Il reste que la Constitution, pour reprendre les propos de P. Alvazzi Del Frate, fait partie de la culture populaire et, en son sein, il existe une conscience de ce que la Constitution est une loi supérieure qui limite le pouvoir.

Cette dimension de la culture comme connaissance de la Constitution, comme elle a été défendue par J. Vachey, doit être relativisée. B. François a précisé que la culture constitutionnelle pouvait être appréhendée en tant que connaissances savantes, à distinguer de la culture populaire, ou en tant que système de représentation, tout en précisant que c'est dans cette seconde dimension qu'il existe une culture constitutionnelle. Il existe un système de représentation par le peuple de la Constitution, celle-ci étant perçue comme un instrument de la culture dominante. A cet égard, l'on ne peut d'ailleurs que constater l'appropriation politique de la Constitution par le politique, l'usage du droit par le politique. En outre, B. François a ajouté que le peuple disposait de compétences en droit constitutionnel, qui s'appuient sur des connaissances et un sentiment de légitimité. Il a renvoyé aux travaux de L. Blondiaux, qui mettent en évidence le fait que ces compétences sont mesurables à partir de la connaissance de l'univers politique, du niveau de cohérence des attitudes et du niveau de stabilité des opinions. Cette compétence présente ainsi une double dimension cognitive et politique, c'est-à-dire, pour cette dernière dimension, d'une aptitude à décider.

La culture constitutionnelle a encore été appréhendée comme une " conscience », une conscience politique (A. Levade) ou une conscience constitutionnelle (B. François, Rafsandjani Hassani Mohamed).

X. Philippe a pris soin de distinguer la culture juridique, la culture politique et la culture constitutionnelle. Rafsandjani Hassani Mohamed, s'il a dénoncé la tendance à l'instrumentalisation de la Constitution par le pouvoir, n'en a pas moins constaté le passage, en Afrique, d'une culture constitutionnelle des armes à une culture constitutionnelle de la Constitution, s'appuyant sur une appropriation de ce texte fondamental par le peuple.

Ces différents éclairages invitent à une réflexion plus personnelle. Il semble exister une représentation par le peuple de la Constitution, à distinguer en l'occurrence de la connaissance savante de ce texte, de ce qu'est la "véritable » Constitution. Cette représentation détermine et conditionne des comportements du peuple (débat, discours, vote, participation à des associations). Autrement dit, c'est moins la Constitution elle-même qui conditionne les comportements du peuple que la représentation que celui-ci en a. En France, le débat autour du projet de loi de ratification du Traité établissant une Constitution pour l'Europe est, sans doute, l'exemple le plus topique d'une dichotomie existante entre le texte sur lequel le peuple devait voter et la représentation de celui-ci, étant entendu que c'est, évidemment, la représentation du texte et non le texte lui-même qui a déterminé les comportements de vote. Autre illustration 
encore, la représentation du droit et de la Constitution dans le cinéma américain est sans doute plus déterminante dans la construction de la culture constitutionnelle américaine et, au-delà, de la culture constitutionnelle américaine perçue par le monde entier, que ne l'est la connaissance universitaire du droit constitutionnel américain.

\section{$\mathbb{S I I}$ - Qu'est-ce que l'on en dit ? Comment développer la culture constitutionnelle?}

La faiblesse supposée ou constatée de la culture constitutionnelle en tant que connaissance a orienté les discours vers la recherche des moyens propres à développer la culture constitutionnelle. Comment peut-on dynamiser la culture constitutionnelle ? Comment peut-on «réinventer la démocratie»? Telles ont été les interrogations lancées par B. François. Le présupposé sur lequel repose cette approche, prescriptive, est que la culture constitutionnelle renforce la qualité de la vie démocratique, tout comme la vigilance du peuple face à l'exercice du pouvoir.

Il existe à cet égard une tension entre la dimension sociale et la dimension politique de la connaissance de la Constitution : dans sa dimension sociale, la connaissance constitutionnelle est perçue comme un savoir des dominants, ce qui va à l'encontre de sa dimension politique, la connaissance constitutionnelle en tant que savoir contre les dominants.

La culture constitutionnelle est une culture de résistance face au pouvoir, le droit constitutionnel étant un droit de l'encadrement du pouvoir, des pouvoirs. Selon cette perspective, difficile d'identifier une culture constitutionnelle en France. Le peuple s'en remet toujours à l'homme providentiel. Quelques dates significatives de consultation directe du peuple : 2 août 1802, 2 août 1804, (10 et 11 décembre 1848), 28 octobre 1962, 27 avril 1969, 24 septembre 2000... trois plébiscites, deux référendums constitutionnels sur le Président de la République et une élection d'un Président bientôt empereur... Une culture constitutionnelle du Chef. C'est encore l'élection présidentielle qui constitue le moment politique du peuple français : 19 décembre 1965, 15 juin 1969, 19 mai 1974, 10 mai 1981, 8 mai 1988, 7 mai 1995, 5 mai 2002, 6 mai 2007, 6 mai 2012, 7 mai 2017. Il n'est d'ailleurs sans doute pas anodin que l'étude IFOP, qui a été évoquée aujourd'hui, visant à apprécier la connaissance de la Constitution par les français, montre que la seule connaissance partagée, non erronée, par tous les sondés était celle de la durée du mandat présidentiel.

Dans la recherche des moyens à utiliser pour développer la culture constitutionnelle, il faut rappeler qu'ils dépendent de la manière dont cette culture est appréhendée : soit en tant que connaissance, soit en tant que compétence. L'on peut ainsi identifier une dimension verticale descendante au phénomène, de la Constitution au peuple, la culture constitutionnelle appréhendée comme une connaissance (A), mais également une dimension verticale ascendante : $\mathrm{du}$ peuple vers la Constitution, la culture constitutionnelle étant entendue comme une compétence (B). 
A - Une dimension verticale descendante: de la Constitution au peuple, la culture constitutionnelle comme connaissance

La Constitution doit aller vers le peuple. P. Alvazzi Del Frate a rappelé l'importance de la connaissance par le peuple de la Constitution afin que se développe, plus largement, une culture juridique de celui-ci. J. Vachey a souligné l'importance de la clarté du texte constitutionnel, de son accessibilité alors que, trop souvent, la Constitution apparait comme un texte « aristocratique ». Il convient ici de rappeler le préambule de la Déclarations des droits de l'homme et du citoyen du 26 août 1789 en vertu duquel : «l'ignorance, l'oubli ou le mépris des droits de l'Homme sont les seules causes des malheurs publics et de la corruption des Gouvernements ».

Défendre l'accessibilité de la Constitution pour le peuple, afin que celui-ci dispose d'une connaissance de celle-ci, s'inscrit dans une logique de réflexivité et d'introspection pour tous ceux qui dispensent le savoir constitutionnel. Cette accessibilité de la Constitution favorise d'ailleurs sa légitimité auprès du peuple. Cette dimension relève, en effet, en partie du moins, de la responsabilité du spécialiste du droit constitutionnel. S’il est vrai que le rôle des institutions, des médias, des think tank et des associations dans la diffusion du droit constitutionnel est important, M. Stefanini a rappelé la place particulière du spécialiste de droit constitutionnel dans cette diffusion. Parce qu'il est spécialiste, sa parole est plus écoutée même si son degré d'influence varie en fonction des auditoires. En tout état de cause, la multiplicité des acteurs de la diffusion ne soulève pas de difficulté dans la mesure où il existe une complémentarité entre les différents acteurs de la diffusion du droit constitutionnel selon J.-P. Dérosier.

Le rôle des enseignants-chercheurs n'en demeure pas moins décisif. Aussi doivent-ils avoir le «soucis de diffuser le savoir sur le droit constitutionnel» selon les mots de J. Vachey. La pédagogie constitutionnelle est importante. P. Alvazzi Del Frate a défendu la mise en place d'un «plan d'éducation nationale» pour la connaissance de la Constitution. J.-Massias a soutenu l'importance du rôle de l'enseignant-chercheur en dehors de l'Université. Celui-ci doit intervenir hors les murs et diffuser la connaissance, en dehors et au-delà du public universitaire. L'on pourrait y voir un rôle de «missionnaire du droit constitutionnel », l'expression est empruntée à D. Maus qui l'avait utilisée à propos du Doyen Favoreu. A. Levade s'est toutefois opposée à la reconnaissance d'un tel rôle, de missionnaire, à la charge de l'universitaire. L'importance de la valorisation des connaissances, de la diffusion des savoirs et de leur vulgarisation a été défendue par J.-P. Massias et M. Stefanini. Dans le prolongement, J.-P. Dérosier a rappelé la nécessité d'adapter les discours en fonction des auditoires. B. François a ajouté que la vulgarisation du propos ne devait pas, pour autant, aboutir à renoncer à la complexité.

M. Stefanini a encore fait appel à l'humilité que devait avoir l'universitaire dans sa démarche visant à rendre accessible son savoir. Cette humilité impose à l'universitaire, comme l'a défendu B. François, de proposer des instruments au peuple plutôt que de prêcher la bonne parole. Il 
n'appartient pas à l'universitaire de dire ce qu'il faut faire : il doit initier des chantiers plutôt que de proposer des constructions toutes faites.

A. Levade convoque l'exemple de la question prioritaire de constitutionnalité (QPC), pour laquelle un effort considérable de diffusion et de communication a été entrepris par le Conseil constitutionnel en particulier et qui est incontestablement un succès : les citoyens (ou, pour le moins, les plaideurs) se sont appropriés la QPC.

Si l'on se tourne du côté des supports de diffusion, B. François a mis en avant, sous l'angle économique du coût des ouvrages, le concept du «prix du kebab», à savoir qu'un ouvrage ne saurait se vendre que s'il ne coûte pas plus cher qu'un kebab. Il plaide pour l'utilisation de tous les supports actuels de diffusion (You Tube, pages Face book). Il a d'ailleurs lui-même un projet de site de droit constitutionnel. Selon ses propres mots, il nous appartient, nous universitaire, de « réenchanter notre métier». J.-P. Dérosier a fait part de son expérience, à propos de son site internet qui est un blog tenu par un universitaire, mais qui n'est pas un blog universitaire sans, pour autant, être un site militant.

$B$ - Une dimension verticale ascendante: $d u$ peuple vers la Constitution, la culture constitutionnelle comme compétence

Il est également possible d'avoir une appréhension de la question de la culture constitutionnelle à partir du peuple. Cette dimension a été mise en évidence essentiellement par B. François. Il a suggéré qu'il fallait sans doute abandonner la logique du citoyen éclairé. Le véritable enjeu ne consiste pas à adapter les citoyens à la démocratie, mais à changer la démocratie pour que le peuple puisse d'y retrouver. Il est nécessaire de faire venir le citoyen à la démocratie.

En outre, B. François a soutenu qu'il était décisif que le citoyen participe de manière véritable à la démocratie, c'est-à-dire que l'intervention du citoyen aboutisse à une décision. Dans un tel contexte, l'on comprend mieux que, si le choix du citoyen à une portée décisionnelle, celui-ci aura un intérêt à acquérir des connaissances de la Constitution. L'effectivité de la participation démocratique nourrit la volonté de connaissance de la démocratie. Dans le prolongement, la pratique constitutionnelle développe la culture constitutionnelle; de la pratique nait la connaissance comme l'a synthétisé B. François, en s'appuyant sur le concept de «compétences pratiques » déjà évoqué.

B. François a encore souligné que l'appropriation par le peuple de la démocratie et, plus particulièrement, de la Constitution, contribue à légitimer le système politique. Il a enfin rappelé que le temps de la culture était un temps long et que le processus de développement de la culture constitutionnelle exigeait du temps. 
A l'issue de cette synthèse, l'on peut être quelque peu rassuré de ce que les juristes peuvent dire d'un objet purement factuel, sans mettre à contribution de données empiriques. Autrement dit, une approche juridique, sans rigueur, d'objets factuels est possible et même éclairante. Peut-être qu'une orientation durable a été, aujourd'hui, fixée à l'initiative de la jeune recherche ; c'est de celle-ci que l'on peut attendre, en principe, le changement. 